\title{
Design and development of a nanoemulsion system containing copper peptide by D- optimal mixture design and evaluation of its physicochemical properties
}

\begin{abstract}
Copper peptide is an important compound used to prevent ageing; a process that leads to several unwanted aging signs such as wrinkles, sagging, pigmented spots and dryness. The Doptimal mixture design was used for optimizing the particle size of Virgin Coconut Oil (VCO) nanoemulsions for topical delivery of copper peptide. Effects of formulation variables including VCO (10ї 20\%, w/w), Tween 80 : Pluronic PF68 (10ї 15\%, w/w), xanthan gum $(0.5 \mathrm{i} 1.0 \%, \mathrm{w} / \mathrm{w})$ and water (64.008ї79.291), towards particle size as a response were studied. D-optimal mixture design analysis demonstrated that the variation in particle size as a response could be expressed as a quadratic function of the main components of the emulsion. Analysis of variance (ANOVA) showed that the quadratic polynomial model sufficiently fits the experimental data. The best combination of the composition factors that gave the optimum particle size was found to be VCO (10\%, w/w), T80 : PF68 (15\%, w/w), xanthan gum $(0.867 \%, \mathrm{w} / \mathrm{w})$ and water $(74.133 \%, \mathrm{w} / \mathrm{w})$. Under these optimum compositions, the actual particle size showed good agreement with predicted particle size with a residual standard error (RSE) less than $2 \%$. The formulation containing copper peptide was successfully prepared with a particle size value of $120.7 \mathrm{~nm}$, which was consistent with the result obtained by TEM. The final formulation also showed good conductivity and $\mathrm{pH}$ values as well as stability against phase separation. A rheology study demonstrates that the VCO nanoemulsion containing copper peptide exhibited shear thinning and pseudoplastic properties and showed more elastic rather than viscous characteristics due to the presence of a large linear viscoelastic region. Permeability test results showed that copper peptide in aqueous solution with and without penetration enhancer cannot be transported through the cellulose acetate membrane after $8 \mathrm{~h}$ application. The nanoemulsion increased the permeability of copper peptide with $21.89 \pm 0.53 \%$ active matter release after $8 \mathrm{~h}$ application.
\end{abstract}

Keyword: D-optimal mixture design; Copper peptide; Virgin Coconut Oil (VCO); Particle size; Nanoemulsion 